\title{
Ergodic Capacity of D2D Underlay Communication using MC-CDMA
}

\author{
Junaid AHMED, Shurjeel WYNE \\ COMSATS University Islamabad, Park Road, Tarlai Kalan, Islamabad, Pakistan \\ junaid@comsats.edu.pk, shurjeel.wyne@comsats.edu.pk
}

Submitted April 24, 2018 / Accepted December 5, 2018

\begin{abstract}
Device to device (D2D) communication that underlay conventional cellular networks can increase their spectrum utilization. However, since D2D pairs share the frequency band with cellular users, interference between these two network tiers can become a major performance bottleneck. In this scenario, use of a spread spectrum technique can be a good choice for D2D communication, due to its inherent interference mitigation capability. In this work, we analyze the achievable ergodic capacity for a D2D user pair that uses multi-carrier code division multiple access (MC-CDMA). Interference from both cellular users and other D2D pairs is considered under Rayleigh faded links and carrier frequency offset. Our derived expression requires a single integration and gives a tight lower bound to achievable ergodic capacity.
\end{abstract}

\section{Keywords}

D2D Communication, ergodic capacity, MC-CDMA, spread spectrum communication, underlay communication

\section{Introduction}

The recent growth of mobile broadband users and the introduction of new applications such as the internet of things (IoT) and machine-to-machine (M2M) communications has resulted in a tremendous increase in mobile data volume. The data traffic is expected to increase further in the coming years, which will worsen the spectrum scarcity and cause overburdening of the cellular infrastructure [1]. Among the technologies being considered for increased spectrum utilization and traffic offloading, direct data exchanges between the users, known as device to device (D2D) communications has emerged as a promising new technology [2]. The D2D communication leverages the short distance typically encountered in IoT and M2M communication to offload traffic from the cellular infrastructure and increase spectrum utilization.

Several methods of D2D communication have been proposed in the literature [3-5]. This includes D2D devices using dedicated resources along with the cellular users in an overlay fashion. In this method, the cellular infrastructure must coordinate between cellular and D2D pairs. This requires additional control signaling that degrades the spectrum utilization and network latency. In the underlay mode of D2D communications, D2D pairs share spectrum with cellular users, this increases the spectrum utilization but may also increase interference to and from the cellular users.

The problem of resource allocation and interference management among D2D pairs has received significant research interest. New interference management and mitigation techniques were proposed in [6], [7]. In [5], intelligent resource allocation was proposed to maximize the achievable throughput. In [8] the authors proposed optimal transmission schemes to maximize the ergodic capacity. In [9-11], the authors proposed the use of advanced mathematical techniques such as nonlinear programming and game theory to maximize throughput.

Spread spectrum techniques such as multi-carrier code division multiple access (MC-CDMA) have inherent interference mitigation capability, nevertheless, they have received little attention in D2D communications. It is well-known that the transmission power of spread spectrum-based D2D pairs remains below the noise floor. Therefore, they cause minimal interference to the orthogonal frequency-division multiple access (OFDMA)-based cellular users (e.g. LTE users in $4 \mathrm{G}$ and $5 \mathrm{G}$ ) in an overlay scheme [12]. Furthermore, the spread spectrum signal of the D2D pairs inherently has high tolerance to narrow band interference caused by the cellular users [12] so that complicated resource allocation techniques for interference mitigation are not required. As such, the spread spectrum techniques require little or no control signaling for coordination and are therefore simpler to implement [13-15].

In [12] and [16], the authors investigated a D2D communication system employing multi-carrier code division multiple access (MC-CDMA). The authors analyzed signal to interference and noise ratio (SINR) and bit error rate performance of the system but did not analyze ergodic capacity. Ergodic capacity of D2D communication has also been analyzed in different scenarios. In [17], ergodic capacity is optimized while minimizing power consumption in a downlink, 
Rician fading environment. In [18] resource allocation to maximize ergodic capacity of a D2D link is analyzed in a vehicle to infrastructure (V2I) scenario. However, no work is reported in the literature that analyze ergodic capacity of spread spectrum techniques in D2D communication. In our work, we analyze the ergodic capacity that can be achieved by D2D pairs employing MC-CDMA while co-existing with cellular users in an underlay mode. Our analysis considers carrier frequency offset impairment at the receiver and interference from both cellular and other D2D pairs under Rayleigh faded links. The derived expression requires a single integration and gives a tight lower bound to ergodic capacity.

The remainder of this paper is organized as follows. Section 2 presents the system model. In Sec. 3 the closedform expressions for ergodic capacity is derived. Section 4 provides the numerical results along with relevant discussion. In Sec. 5, some concluding remarks are given.

\section{System Model}

Consider D2D communication underlaying a cellular network. Cellular users communicate with the base station using OFDMA (e.g. LTE, WiMax etc), while the D2D pairs communicate using MC-CDMA and share the available frequency band. Let the transmitted symbol of a typical D2D user be given as

$$
s(t)=\frac{1}{\sqrt{N_{p}}} \sum_{n=0}^{N_{p}-1} b_{d}(t) c_{d}[n] \mathrm{e}^{\mathrm{j} 2 \pi f_{n} t}
$$

where $b_{d}(t)$ and $c_{d}[n]$ are the $d$-th transmitter's information bearing signal and spreading code, respectively, we assume $c_{d}[n] \in\{-1,1\}$ with equal probability. $N_{p}$ is the number of subcarriers used by each D2D user and $f_{n}$ represents frequency of the $n$-th subcarrier. Let there be $K+1$ D2D pairs and $C$ cellular users, where each cellular user uses $N_{c}$ subcarriers. This means that $C \times N_{c}$ subcarriers are used by the cellular users. For ease of analysis, and without loss of accuracy, the system is equivalently modeled as $L=C \times N_{c}$ single-carrier cellular users. Let there be a pair-wise power control mechanism such that the received signal power at every D2D receiver is equal, and set to unity without loss of generality. The transmitted signal is subjected to slow-flat multipath Rayleigh fading channel and distance-dependent pathloss. The channel gain of the n-th subcarrier due to fading is $G_{n}=\sum_{l=0}^{Z-1} g_{l} \mathrm{e}^{-\mathrm{j} 2 \pi f_{n} \tau_{l}}$, where $g_{l}$ and $\tau_{l}$ are the complex amplitude and propagation delay of the $l$-th path, where $l \in[0, Z-1]$. We assume that all $g_{l}$ are zero mean uncorrelated complex Gaussian random variables with normalized power such that $\sum_{l=0}^{Z-1} \mathbb{E}\left[\left|g_{l}\right|^{2}\right]=1$, where $\mathbb{E}$ is the expectation operator. Therefore $\left\{G_{0}, \ldots, G_{N_{p}-1}\right\}$ are zero mean complex Gaussian random variables with unit variance. The received signal at the D2D receiver of interest can then be written as

$$
\begin{aligned}
R(t) & =\frac{1}{\sqrt{N_{p}}} \sum_{n=0}^{N_{p}-1} G_{n} b_{d}(t) c_{d}[n] \mathrm{e}^{\mathrm{j} 2 \pi f_{n} t} \\
& +\sum_{k=1}^{K} \frac{\sqrt{r_{k}^{-\beta}}}{\sqrt{N_{p}}} \sum_{n=0}^{N_{p}-1} G_{n} b_{k}(t) c_{k}[n] \mathrm{e}^{\mathrm{j} 2 \pi f_{n} t} \mathrm{e}^{\mathrm{j} 2 \pi \frac{\Delta_{k}}{T} t} \\
& +\sum_{n=0}^{L-1} \sqrt{\alpha r_{n}^{-\beta}} G_{n} b_{n}(t) c_{n}[n] \mathrm{e}^{\mathrm{j} 2 \pi f_{n} t} \mathrm{e}^{\mathrm{j} 2 \pi \frac{\Delta n}{T} t}+\eta(t)
\end{aligned}
$$

where $r_{k}$ is the distance between the $k$-th transmitter and the $\mathrm{D} 2 \mathrm{D}$ receiver of interest, and $\beta$ is the pathloss exponent. Furthermore, $\Delta_{k}$ is the normalized carrier frequency offset between the $k$-th transmitter and the receiver of interest, and $T$ is the symbol duration. In (2), the first term is desired signal component, the second term is interference from other D2D transmitters, the third term is interference from the cellular users, and the fourth term $\eta(t)$ is due to noise that is assumed to be Gaussian with two sided power spectral density of $N_{0}$. The D2D receiver is assumed to perfectly estimate and compensate the frequency offset, so that, it does not appear in the desired signal component. In (2), $\alpha$ is a ratio between average power transmitted on a single subcarrier by a cellular user and the average transmit power of a D2D transmitter. Since cellular users are expected to transmit at a higher power level than D2D transmitters, in the considered system model $\alpha \geq 1$. The received signal is decoded by a conventional correlation receiver with maximum ratio combining, resulting in a decision variable $Z$ given as

$$
Z=\sum_{n=0}^{N_{p}-1} \frac{1}{T} \int_{0}^{T} G_{n}^{*} c[n] \mathrm{e}^{-\mathrm{j} 2 \pi f_{n} t} R(t) \mathrm{d} t .
$$

The decision variable can be separated into four components: a desired signal $S$, interference from other D2D pairs $I_{\mathrm{D}}$, interference from the cellular users $I_{\mathrm{C}}$ and noise $N$. These components can be individually written as

$$
\begin{gathered}
S=\frac{1}{\sqrt{N_{p}}} \sum_{n=0}^{N_{p}-1} \frac{1}{T} \int_{0}^{T} b(t)\left|G_{n}\right|^{2} \mathrm{~d} t, \\
I_{\mathrm{D}}=\sum_{m=0}^{N_{p}-1} \int_{0}^{T} G_{m}^{*} c[m] \mathrm{e}^{-\mathrm{j} 2 \pi f_{m} t} \sum_{k=1}^{K} \frac{\sqrt{r_{k}^{-\beta}}}{\sqrt{N_{p}}} \sum_{n=0}^{N_{p}-1} G_{n} \\
\times b_{k}(t) c_{k}[n] \mathrm{e}^{\mathrm{j} 2 \pi f_{n} t} \mathrm{e}^{\mathrm{j} 2 \pi \frac{\Delta k}{T} t} \mathrm{~d} t, \\
I_{\mathrm{C}}=\sum_{m=0}^{N_{p}-1} \int_{0}^{T} G_{m}^{*} c[m] \mathrm{e}^{-\mathrm{j} 2 \pi f_{m} t} \sum_{n=0}^{L-1} \sqrt{\alpha r_{n}^{-\beta}} G_{n} b_{n}(t) \\
\times c_{n}[n] \mathrm{e}^{\mathrm{j} 2 \pi f_{n} t} \mathrm{e}^{\mathrm{j} 2 \pi \frac{\Delta n}{T} t} \mathrm{~d} t,
\end{gathered}
$$




$$
N=\sum_{m=0}^{N_{p}-1} G_{m}^{*} c[m] \frac{1}{T} \int_{0}^{T} \mathrm{e}^{-\mathrm{j} 2 \pi f_{m} t} \eta(t) \mathrm{d} t .
$$

This noise component $N$ in the test-statistic $\mathrm{Z}$ provided by the decoder at its output is due to $\eta(t)$ in the received signal waveform $R(t)$, its variance is $N_{0}$. Since $Z$ is sum of a large number of independent random variables, its distribution can be assumed Gaussian in light of the central limit theorem. The conditional mean of $Z$ is given as

$$
\mathbb{E}\left[Z \mid G_{0}, \ldots, G_{N_{p}-1}\right]=\sqrt{\frac{E_{\mathrm{s}}}{T N_{p}}} \sum_{n=0}^{N_{p}-1}\left|G_{n}\right|^{2}
$$

where $E_{\mathrm{s}}$ is the symbol energy. This conditional mean has a contribution from $S$ only, because the interference and noise terms are zero mean. On the other hand, the conditional variance has contributions from interference and noise terms only, because we are conditioning on all random variable in $S$. Using (4)-(8), the instantaneous SINR at the D2D receiver of interest can be expressed as

$$
\begin{gathered}
\text { SINR }=\frac{E_{\mathrm{s}}}{T N_{p}}\left(\sum_{n=0}^{N_{p}-1}\left|G_{n}\right|^{2}\right)^{2}\left[\frac{E_{\mathrm{s}}}{T N_{p}} \sum_{k=1}^{K} r_{k}^{-\beta} \sum_{m=0}^{N_{p}-1}\right. \\
\times \sum_{n=0}^{N_{p}-1} \frac{1-\operatorname{sinc}\left(2 \pi\left(m-n+\Delta_{k}\right)\right)}{\left(\pi\left(m-n+\Delta_{k}\right)\right)^{2}}\left|G_{m}\right|^{2}\left|G_{n}\right|^{2} \\
+\frac{\alpha E_{\mathrm{s}}}{T N_{c}} \sum_{n=0}^{L N_{c}-1} r_{n}^{-\beta} \sum_{m=0}^{N_{p}-1} \frac{1-\operatorname{sinc}\left(2 \pi\left(m-n+\Delta_{n}\right)\right)}{\left(\pi\left(m-n+\Delta_{n}\right)\right)^{2}} \\
\left.\times\left|G_{m}\right|^{2}\left|G_{n}\right|^{2}+\sum_{m=0}^{N_{p}-1}\left|G_{m}\right|^{2} \frac{N_{0}}{T}\right]^{-1} .
\end{gathered}
$$

Let $\rho_{k, m, n}=\frac{1-\operatorname{sinc}\left(2 \pi\left(m-n+\Delta_{k}\right)\right)}{\left(\pi\left(m-n+\Delta_{k}\right)\right)^{2}}$ and $\chi_{k, n}=\sum_{m=0}^{N_{p}-1} \frac{\left|G_{m}\right|^{2}}{\sum_{\bar{m}=0}^{N_{p}-1}\left|G_{\bar{m}}\right|^{2}} \rho_{k, m, n}$, (9) can be further simplified as

$$
\begin{aligned}
\operatorname{SINR}= & \sum_{n=0}^{N_{p}-1}\left|G_{n}\right|^{2}\left[\sum_{k=1}^{K} r_{k}^{-\beta} \sum_{n=0}^{N_{p}-1} \chi_{k, n}\left|G_{n}\right|^{2}\right. \\
& \left.+\alpha \frac{N_{p}}{N_{c}} \sum_{n=0}^{L N_{c}-1} r_{n}^{-\beta} \chi_{n, n}\left|G_{n}\right|^{2}+N_{p} \frac{N_{0}}{E_{\mathrm{S}}}\right]^{-1} .
\end{aligned}
$$

Using the instantaneous SINR expression in (10), the ergodic capacity of the D2D pair can be found using Shannon's capacity formula $C=\mathbb{E}\left[\log _{2}(1+S I N R)\right]$. However, a direct computation of the expectation involves $(K+1) \times N_{p}+L \times N_{c}$ simultaneous integrations, making this approach intractable. In the next section, we attempt to simplify the capacity expression.

\section{Ergodic Capacity Analysis}

The ergodic capacity of a D2D pair in the presence of $K$ other D2D pairs and $L$ cellular users can be expressed, using (10) as

$$
\begin{aligned}
C=\mathbb{E} & {\left[\operatorname { l o g } _ { 2 } \left(1+\sum_{n=0}^{N_{p}-1}\left|G_{n}\right|^{2}\left[\sum_{k=1}^{K} r_{k}^{-\beta} \sum_{n=0}^{N_{p}-1} \chi_{k, n}\left|G_{n}\right|^{2}\right.\right.\right.} \\
+\alpha & \left.\left.\left.\frac{N_{p}}{N_{c}} \sum_{n=0}^{L N_{c}-1} r_{n}^{-\beta} \chi_{n, n}\left|G_{n}\right|^{2}+N_{p} \frac{N_{0}}{E_{\mathrm{s}}}\right]^{-1}\right)\right]
\end{aligned}
$$

It can be easily shown that $\log _{2}(1+S I N R)$ is convex as its second derivative is strictly non-negative for all positive values of SINR. Therefore, Jensen's inequality can be applied on (11) to obtain

$$
\begin{aligned}
C \geq \mathbb{E}\left[\operatorname { l o g } _ { 2 } \left(1+\sum_{n=0}^{N_{p}-1}\left|G_{n}\right|^{2}\left[\sum_{k=1}^{K} \sum_{n=0}^{N_{p}-1} \hat{\chi}_{k, n}\left|G_{n}\right|^{2}\right.\right.\right. \\
\left.\left.\left.+\alpha \frac{N_{p}}{N_{c}} \sum_{n=0}^{L N_{c}-1} \hat{\chi}_{n, n}\left|G_{n}\right|^{2}+N_{p} \frac{N_{0}}{E_{\mathrm{s}}}\right]^{-1}\right)\right]
\end{aligned}
$$

where $\hat{\chi}_{k, n}=\mathbb{E}\left[r_{k}^{-\beta} \chi_{k, n}\right]$, since $r_{k}$ and $\chi_{k, n}$ are independent $\hat{\chi}_{k, n}=\mathbb{E}\left[r_{k}^{-\beta}\right] \mathbb{E}\left[\chi_{k, n}\right]=\frac{2\left(a^{2-\beta}-R^{2-\beta}\right)}{(-2+\beta) R^{2}} \mathbb{E}\left[\chi_{k, n}\right]$ where $a$ and $R$ are minimum and maximum values of $r_{k}$ respectively. We used the pdf of $r_{k}$ as $f_{r_{k}}(r)=2 r /\left(R^{2}-a^{2}\right)$, which can be easily proved. Solution to $\mathbb{E}\left[\chi_{k, n}\right]$ is given in Appendix in [19]. Using Lemma 1 in [20] (12) can be written as

$$
\begin{array}{r}
C \geq \log _{2} \mathrm{e} \int_{0}^{\infty} \frac{1}{z}\left(\mathbb { E } \left[\operatorname { e x p } \left(-z\left(\sum_{k=1}^{K} \sum_{n=0}^{N_{p}-1} \hat{\chi}_{k, n}\left|G_{n}\right|^{2}\right.\right.\right.\right. \\
\left.\left.\left.+\alpha \frac{N_{p}}{N_{c}} \sum_{n=0}^{L N_{c}-1} \hat{\chi}_{n, n}\left|G_{n}\right|^{2}\right)\right)\right]-\mathbb{E}\left[\operatorname { e x p } \left(-z\left(\sum_{k=1}^{K} \sum_{n=0}^{N_{p}-1}\right.\right.\right. \\
\left.\left.\left.\left.\times \hat{\chi}_{k, n}\left|G_{n}\right|^{2}+\alpha \frac{N_{p}}{N_{c}} \sum_{n=0}^{L N_{c}-1} \hat{\chi}_{n, n}\left|G_{n}\right|^{2}+\sum_{n=0}^{N_{p}-1}\left|G_{n}\right|^{2}\right)\right)\right]\right) \\
\times \mathrm{e}^{-z \frac{N_{0} N_{p}}{E_{\mathrm{s}}}} \mathrm{d} z .
\end{array}
$$

In (13), the terms in the exponents are independent, hence the expectations can be taken separately. Furthermore, summations in the exponents can be written as multiplications of exponentials, therefore (13) can also be written as 


$$
\begin{aligned}
& C \geq \log _{2} \mathrm{e} \int_{0}^{\infty} \frac{1}{z}\left(\prod_{k=1}^{K} \mathbb{E}\left[\mathrm{e}^{-z \sum_{n=0}^{N_{p}-1} \hat{\hat{\chi}_{k, n}}\left|G_{n}\right|^{2}}\right]\right. \\
& \times \prod_{n=0}^{L N_{c}-1} \mathbb{E}\left[\mathrm{e}^{-z \alpha \frac{N_{p}}{N_{c}} \hat{\chi}_{n, n}\left|G_{n}\right|^{2}}\right]-\prod_{k=1}^{K} \mathbb{E}\left[\mathrm{e}^{-z \sum_{m=0}^{N_{p}-1} \hat{\chi}_{k, n}\left|G_{n}\right|^{2}}\right] \\
& \left.\times \prod_{n=0}^{L N_{c}-1} \mathbb{E}\left[\mathrm{e}^{-z \alpha \frac{N_{p}}{N_{c}} \hat{\chi}_{n, n}\left|G_{n}\right|^{2}}\right] \mathbb{E}\left[\mathrm{e}^{-z \sum_{n=0}^{N_{p}-1}\left|G_{n}\right|^{2}}\right]\right) \\
& \times \mathrm{e}^{-z \frac{N_{0} N p}{E_{\mathrm{s}}}} \mathrm{d} z \text {. }
\end{aligned}
$$

Using (4a) in [21], i.e. MGF of Gaussian quadratic forms, (14) can be written as

$$
\begin{aligned}
C \geq \log _{2} \mathrm{e} \int_{0}^{\infty} \frac{1}{z} & \left(\frac{1}{\left|\mathbf{I}_{N_{p}}+z \boldsymbol{\Lambda}\right|}\right)^{K}\left(\frac{1}{\left|\mathbf{I}_{L}+z \alpha N_{p} \boldsymbol{\Lambda}\right|}\right) \\
& \times\left(1-\frac{1}{\left|\mathbf{I}_{N_{p}}+z \boldsymbol{\Lambda}^{\mathbf{1}}\right|}\right) \mathrm{e}^{-z \frac{N_{0} N_{p}}{E_{\mathrm{S}}}} \mathrm{d} z,
\end{aligned}
$$

where $\boldsymbol{\Lambda}(i, j)=\mathbb{E}\left[G_{i, k} G_{j, k}^{*} \sqrt{\hat{\chi}_{k, i} \hat{\chi}_{k, j}}\right]$ and $\boldsymbol{\Lambda}^{\mathbf{1}}(i, j)=$ $\mathbb{E}\left[G_{i, k} G_{j, k}^{*}\right], \mid$.| represents determinant of a matrix. Equation (15) is the desired expression for lower bound to ergodic capacity of a D2D link in the presence of other D2D pairs and cellular users in a Rayleigh fading environment. It requires a single integration as compared to $(K+1) \times N_{p}+L$ integration's originally.

\section{Numerical Results}

In this section we provide simulation results to validate the analysis. Monte Carlo method was used to validate the lower bound to ergodic capacity expression in (15), where SINR is given in (10). Simulation parameters are given in Tab. 1.

In Fig. 1, the ergodic capacity is plotted against $E_{\mathrm{S}} / N_{0}$, it shows a steady increase in the ergodic capacity of the D2D pairs with increasing $\operatorname{SNR}\left(E_{\mathrm{s}} / N_{0}\right)$. There is also a significant increase in the capacity when the number of subcarriers used by D2D pairs $N_{p}$ is increased. This is because spreading the signal on a larger number of subcarriers increases its ability to combat fading and interference from both cellular and other D2D pairs. The simulation results closely follow the analytical plots, confirming the validity of the presented analysis. Note that even at $0 \mathrm{~dB}, \mathrm{D} 2 \mathrm{D}$ pairs are able to achieve ergodic capacity of $1 \mathrm{bit} / \mathrm{sec} / \mathrm{Hz}$, which is quite significant. This is due to the inherent tolerance of MC-CDMA to interference. This makes MC-CDMA a good choice for D2D communication when device coordination for resource allocation etc is not possible.

Figure 2 shows the reduction in ergodic capacity with an increasing number of cellular users $L$, while the number of D2D pairs $K$ is kept constant. It was generated for $E_{\mathrm{s}} / N_{0}$ value of $20 \mathrm{~dB}$. Since the cellular users are a major source of interference for the D2D pairs, an increase in $L$ results in more interference and hence the drop. This reduction is more pronounced for a higher value of $\alpha$, which corresponds to higher transmit power of the cellular user, per subcarrier, relative to a D2D user. The reduction in capacity is around 20\% for $\alpha=1$ and around $50 \%$ for $\alpha=5$, when $L$ is increased from 0 i.e. no cellular user to 256 i.e. maximum possible cellular users. Therefore, it is apparent that D2D communication is still possible, albeit at a slower rate, even when maximum possible cellular users are active. This is because a relatively large spreading factor $N_{p}$ of 256 is used for the D2D pairs. Similar results are shown in Fig. 3, where ergodic capacity is plotted against number of D2D pairs $K$, while keeping the number of cellular users constant. There is a reduction in the $\mathrm{D} 2 \mathrm{D}$ capacity with an increase in $K$, due to the increasing interference from new D2D pairs, however, the overall effect is not significant (around 13\%). This also shows the benefit of using MC-CDMA for D2D pairs in combating interference from both cellular and other D2D pairs.

In Fig. 4, ergodic capacity is plotted against the cell radius. In order to keep the comparison fair, the number of D2D pairs is steadily increased with increasing $R$, while keeping a constant D2D user density and $L$. It can be seen that the capacity initially increases with cell size. However, after a certain size the increase becomes insignificant. This is because in larger cells the users are more spread out and hence offer lesser interference due to the pathloss factor. This shows that small cell size has little benefit and increasing cell size results in capacity gain, however, this gain has a diminishing return with cell size.

\begin{tabular}{|c|c|}
\hline Simulation Parameters & Value \\
\hline \hline Number of cellular users $(L)$ & 16 \\
\hline Pathloss exponent $(\beta)$ & 4 \\
\hline $\begin{array}{c}\text { Single subcarrier power ratio, } \\
\text { cellular/D2D user }(\alpha)\end{array}$ & 5 \\
\hline Maximum cell size $(R)$ & $500 \mathrm{~m}$ \\
\hline Number of D2D pairs & 10 \\
\hline$E_{\mathrm{s}} / N_{0}$ & $20 \mathrm{~dB}$ \\
\hline Number of subcarriers $\left(N_{p}\right)$ & 256 \\
\hline Channel delay spread & $0.1 \mu \mathrm{s}$ \\
\hline $\begin{array}{c}\text { Frequency separation between } \\
\text { adjacent subcarriers }\end{array}$ & $312.5 \mathrm{kHz}$ \\
\hline Simulation iterations & $10^{4}$ \\
\hline
\end{tabular}

Tab. 1. Simulation parameters. 


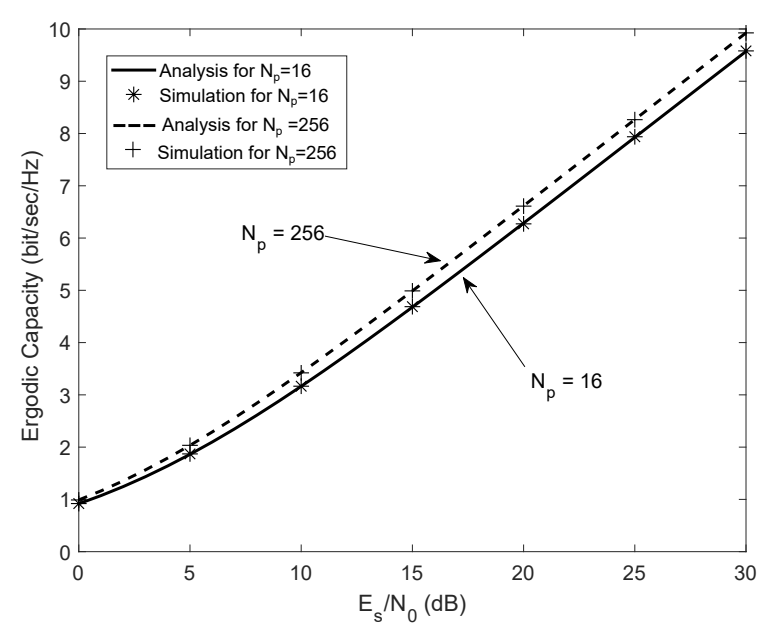

Fig. 1. Ergodic capacity increase versus $E_{\mathrm{s}} / N_{0}$ for some spreading factors.

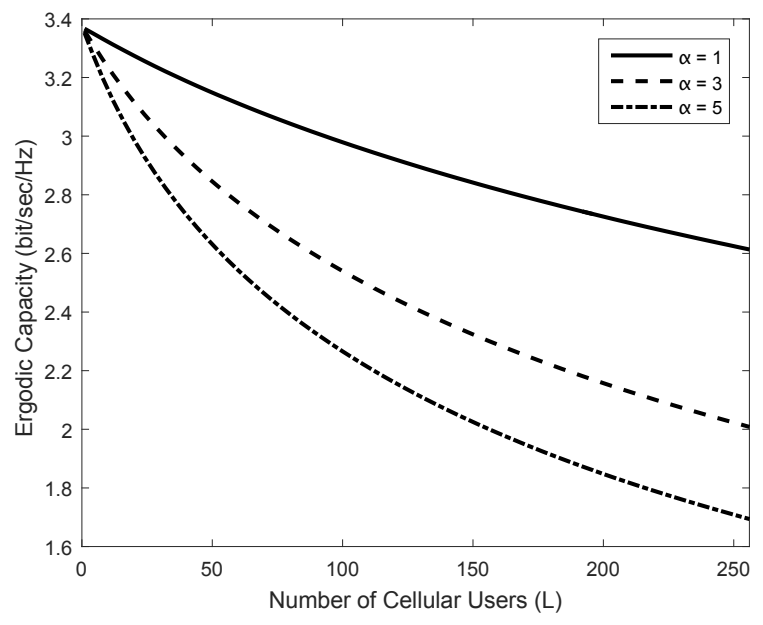

Fig. 2. Ergodic capacity versus number of cellular users for some $\alpha$ values at $E_{S} / N_{0}=20 \mathrm{~dB}$

\section{Conclusion}

We have derived a novel expression for lower bound to ergodic capacity of a D2D communication pair that uses MC-CDMA and operates in underlay mode with a cellular network. Our expression is computationally efficient and can be used to analyze the achievable ergodic capacity, in a variety of interference scenarios. Our results show that MC-CDMA can be used for D2D communication underlying cellular networks. High ergodic capacity is achieved as MCCDMA is resilient to interference from both cellular users and other D2D pairs. Furthermore, it requires little or no overhead for resource allocation, thus its implementation is simple. It is also shown that larger cell sizes give higher ergodic capacity, however, this gain has a diminishing return with cell size. This work can be extended in future by considering a heterogeneous network of base stations.

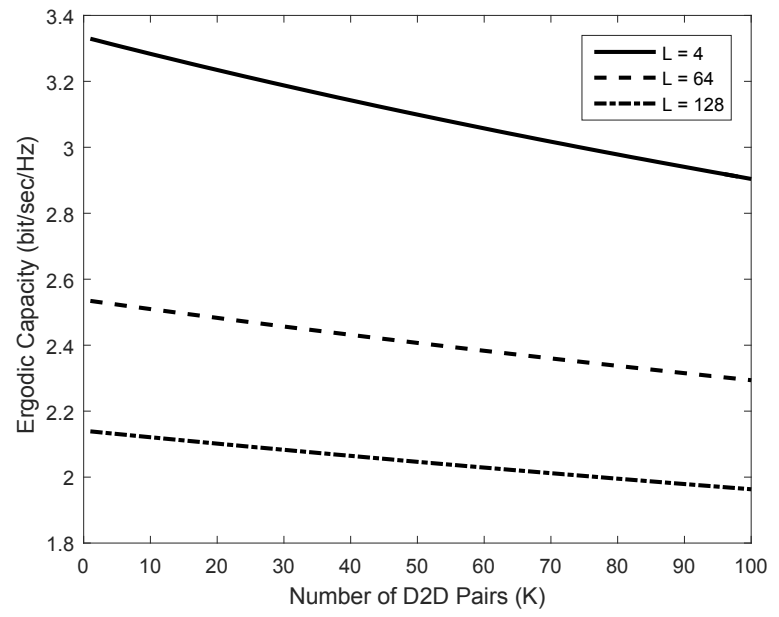

Fig. 3. Ergodic capacity versus number of D2D pairs for some $L$ values.

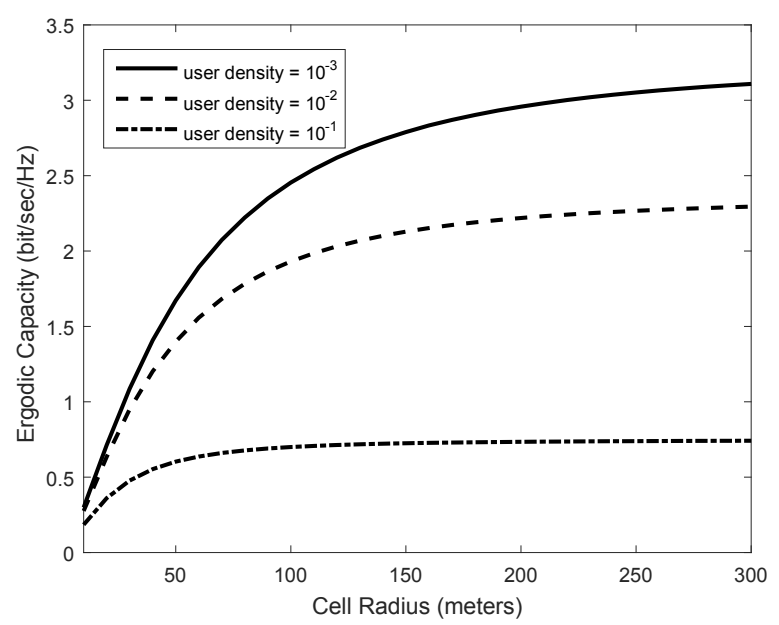

Fig. 4. Ergodic capacity versus cell radius $(R)$ for some user densities.

\section{References}

[1] TEHRANI, M. N., UYSAL, M., YANIKOMEROGLU, H. Deviceto-device communication in $5 \mathrm{G}$ cellular networks: challenges, solutions, and future directions. IEEE Communications Magazine, 2014, vol. 52, no. 5, p. 86-92. DOI: 10.1109/MCOM.2014.6815897

[2] LIEN, S. Y., CHIEN, C. C., TSENG, F. M., HO, T. C. 3GPP device-to-device communications for beyond $4 \mathrm{G}$ cellular networks. IEEE Communications Magazine, 2016, vol. 54, no. 3, p. 29-35. DOI: 10.1109/MCOM.2016.7432168

[3] ASADI, A., WANG, Q., MANCUSO, V. A survey on deviceto-device communication in cellular networks. IEEE Communications Surveys Tutorials, 2014, vol. 16, no. 4, p. 1801-1819. DOI: 10.1109/COMST.2014.2319555

[4] CAO, Y., JIANG, T., WANG, C. Cooperative device-to-device communications in cellular networks. IEEE Wireless Communications, 2015, vol. 22, no. 3, p. 124-129. DOI: 10.1109/MWC.2015.7143335 
[5] MESHGI, H., ZHAO, D., ZHENG, R. Optimal resource allocation in multicast device-to-device communications underlaying LTE networks. IEEE Transactions on Vehicular Technology, 2017, vol. 66 , no. 9, p. 8357-8371. DOI: 10.1109/TVT.2017.2691470

[6] MIN, H., LEE, J., PARK, S., HONG, D. Capacity enhancement using an interference limited area for device-to-device uplink underlaying cellular networks. IEEE Transactions on Wireless Communications, 2011, vol. 10, no. 12, p. 3995-4000. DOI: 10.1109/TWC.2011.100611.101684

[7] KWON, H., LEE, J., KANG, I. Interference-aware interference mitigation for device-to-device communications. In Proceedings of the IEEE Vehicular Technology Conference (VTC Spring). Seoul (South Korea), 2014, p. 1-5. DOI: 10.1109/VTCSpring.2014.7022956

[8] LEE, D., KIM, S.-I., LEE, J., HEO, J. Power allocation and transmission period selection for device-to-device communication as an underlay to cellular networks. Wireless Personal Communications, 2014, vol. 79, no. 1, p. 1-20. DOI: $10.1007 /$ s11277-014-1837-5

[9] YIN, R., YU, G., ZHONG, C., ZHANG, Z. Distributed resource allocation for D2D communication underlaying cellular networks. In Proceedings of the IEEE International Conference on Communications Workshops (ICC). Budapest (Hungary), 2013, p. 138-143. DOI: 10.1109/ICCW.2013.6649216

[10] LEE, D. H., CHOI, K. W., JEON, W. S., JEONG, D. G Two-stage semi-distributed resource management for device-todevice communication in cellular networks. IEEE Transactions on Wireless Communications, 2014, vol. 13, no. 4, p. 1908-1920. DOI: 10.1109/TWC.2014.022014.130480

[11] SONG, L., NIYATO, D., HAN, Z., HOSSAIN, E. Game-theoretic resource allocation methods for device-to-device communication. IEEE Wireless Communications, 2014, vol. 21, no. 3, p. 136-144. DOI: 10.1109/MWC.2014.6845058

[12] XING, H., RENFORS, M. Multi-carrier CDMA for network assisted device-to-device communications for an integrated OFDMA cellular system. In Proceedings of the IEEE Vehicular Technology Conference (VTC Spring). Nanjing (China), 2016, p. 1-6. DOI: 10.1109/VTCSpring.2016.7504354

[13] AHMED, J., HAMDI, K. Spectral efficiency degradation of multicarrier CDMA due to carrier frequency offset. In Proceedings of the IEEE International Conference on Communications (ICC). Kyoto (Japan), 2011, p. 1-5. DOI: 10.1109/icc.2011.5963490

[14] AHMED, J. Spectral efficiency comparison of OFDM and MC-CDMA with carrier frequency offset. Radioengineering, 2017, vol. 26, no. 1, p. 221-226. DOI: 10.13164/re.2017.0221

[15] AHMED, J., TIWANA, M. I., AHMED, O., SOHAIB, S. Energy and area spectral efficiency trade-off for mc-cdma with carrier frequency offset. Turkish Journal of Electrical Engineering \& Computer Sciences, 2017, vol. 25, no. 4, p. 3052-3060. DOI: $10.3906 / \mathrm{elk}-1608-285$
[16] XING, H., RENFORS, M. Link level performance of a multicarrier CDMA based device-to-device integrated OFDMA cellular system. In Proceedings of the European European Wireless Conference. Oulu (Finland), 2016, p. 1-6.

[17] LIU, C., NATARAJAN, B. Power-aware maximization of ergodic capacity in D2D underlay networks. IEEE Transactions on Vehicular Technology, 2017, vol. 66, no. 3, p. 2727-2739. DOI: $10.1109 /$ TVT.2016.2583960

[18] LIANG, L., LI, G. Y., XU, W. Resource allocation for D2D-enabled vehicular communications. IEEE Transactions on Communications, 2017, vol. 65, no. 7, p. 3186-3197. DOI: 10.1109/TCOMM.2017.2699194

[19] AHMED, J., HAMDI, K. Spectral efficiency of asynchronous MC-CDMA with frequency offset over correlated fading. IEEE Transactions on Vehicular Technology, 2013, vol. 62, no. 7, p. 3423-3429. DOI: 10.1109/TVT.2013.2253339

[20] HAMDI, K. A useful lemma for capacity analysis of fading interference channels. IEEE Transactions on Communications, 2010, vol. 58, no. 2, p. 411-416. DOI: 10.1109/TCOMM.2010.02.080117

[21] TURIN, G. L. The characteristic function of Hermitian quadratic forms in complex normal variables. Biometrika, 1960, vol. 47, p. 199-201. DOI: $10.2307 / 2332977$

\section{About the Authors...}

Junaid AHMED is Assistant Professor at COMSATS University Islamabad, Pakistan. He graduated from Middle East Technical University in Ankara, Turkey in 1997. His MSc degree is from Oklahoma State University, Stillwater, Oklahoma, USA and Ph.D. degree from The University of Manchester, UK in 2012. His research interests include wireless communications and networks, and 5G cellular communications.

Shurjeel WYNE (S'02 - M'08 - SM'13) received his Ph.D. from Lund University, Sweden in March 2009. Between April 2009 and April 2010, he was a Post-Doctoral Research Fellow funded by the High-Speed Wireless Centre at Lund University. Since June 2010 he is with the Department of Electrical Engineering at COMSATS University Islamabad, Pakistan, where he serves as an Associate Professor. His research interests include wireless channel characterization and modeling, cooperative communications, physical layer security, and wireless information and power transfer. He is a Co-recipient of the best paper award of the Antennas and Propagation Track in IEEE VTC2013-Spring. 